MATHEMATICS OF COMPUTATION

Volume 70, Number 235, Pages 1043-1069

S 0025-5718(00)01254-0

Article electronically published on May 23, 2000

\title{
EXISTENCE OF DISCRETE SHOCK PROFILES OF A CLASS OF MONOTONICITY PRESERVING SCHEMES FOR CONSERVATION LAWS
}

\author{
HAITAO FAN
}

\begin{abstract}
When shock speed $s$ times $\Delta t / \Delta x$ is rational, the existence of solutions of shock profile equations on bounded intervals for monotonicity preserving schemes with continuous numerical flux is proved. A sufficient condition under which the above solutions can be extended to $-\infty<j<\infty$, implying the existence of discrete shock profiles of numerical schemes, is provided. A class of monotonicity preserving schemes, including all monotonicity preserving schemes with $C^{1}$ numerical flux functions, the second order upwinding flux based MUSCL scheme, the second order flux based MUSCL scheme with LaxFriedrichs' splitting, and the Godunov scheme for scalar conservation laws are found to satisfy this condition. Thus, the existence of discrete shock profiles for these schemes is established when $s \Delta t / \Delta x$ is rational.
\end{abstract}

\section{INTRODUCTION}

The field equations expressing the balance laws for one-dimensional homogeneous continuous media typically have the form of systems of conservation laws

$$
u_{t}+f(u)_{x}=0, \quad x \in \mathbb{R}, \quad t>0, \quad u \in \mathbb{R}^{n}
$$

In this paper, we shall investigate the existence of discrete shock profiles of some monotonicity preserving schemes, including second order flux based MUSCL schemes, for scalar conservation laws. We denote these schemes by

$$
u_{j}^{n+1}=u_{j}^{n}-\lambda\left(\bar{f}_{j+1 / 2}^{n}-\bar{f}_{j-1 / 2}^{n}\right),
$$

where $\lambda:=\Delta t / \Delta x$ and $\left|\lambda f^{\prime}\right|<1$. The numerical flux function $\bar{f}$ satisfies the consistency condition that $\bar{f}$ is continuous and

$$
\bar{f}(u, u, \ldots, u)=f(u) .
$$

We denote speeds of shock profiles of (1.2) by $s$.

It is well known that in general the solution of the initial value problem of (1.1) develops discontinuities in a finite time which present difficulties for numerical computation of solutions of (1.1). Shock profiles of numerical schemes for (1.1) epitomize the propagation and structure properties of shocks in numerical solutions. It is also closely related to error estimates of the numerical solutions near shocks

Received by the editor October 23, 1998 and, in revised form, July 23, 1999.

2000 Mathematics Subject Classification. Primary 80A32, 35L65, 35L67.

Key words and phrases. Discrete shock profile, discrete traveling wave, monotonicity preserving scheme, MUSCL scheme, conservation laws.

Research supported by NSF Fellowship under Grant DMS-9306064. 
(see $[\mathrm{EY}],[\mathrm{F}], \mathrm{Je}, \mathrm{LX}],[\mathrm{TT}],[\mathrm{TZ}]$ ). Thus, a brief review of the known results on the existence discrete shock profiles of numerical schemes for conservation laws is in order. Jennings [Je] proved the existence and stability of discrete traveling waves for strictly monotone schemes with differentiable fluxes for scalar conservation laws. The existence of discrete shock waves of first order accurate finite difference schemes for systems of conservation laws when $\lambda s$ is rational was established by Majda and Ralston [MR] by using the center manifold theorem (see also [Mi]). Yu [Yu proved the existence of discrete shocks for the Lax-Wendroff scheme when $\lambda s$ is rational or $s$ is small. Fan $[\mathrm{F}$ ] established the existence and uniqueness of the Lipschitz continuous shock profile for Godunov scheme.

Almost all of above results are either for monotone schemes or for the LaxWendroff scheme. On the other hand, almost all useful high resolution schemes are adaptive and few analytical results involving shocks are available for these schemes, even though some of them are widely used. Some of the major difficulties in analyzing these schemes are their adaptiveness and that their flux functions $\bar{f}_{j+1 / 2}$ are at most Lipschitz continuous rather than continuously differentiable.

In this paper, we consider the existence of discrete shock profiles for some adaptive monotonicity preserving schemes for scalar conservation laws. A scheme (1.2) is called monotonicity preserving if the monotonicity of $u^{n}$ implies the same type of monotonicity of $u^{n+1}$. Monotonicity preserving schemes include monotone schemes, $l^{1}$-contracting schemes and TVD schemes. Many well known numerical schemes are monotonicity preserving schemes. For example, the Lax-Friedrichs' scheme is monotone, the Godunov's scheme is strictly $l^{1}$-contracting and many second order MUSCL schemes are TVD, and hence they are monotonicity preserving schemes. We assume the schemes satisfy the following condition which is equivalent to that the monotonicity preserving property of schemes is kept when $\Delta t$ becomes smaller:

Assumption I. The scheme (1.2) is monotonicity preserving for all $0<\lambda \leq \lambda_{0}$ for some $\lambda_{0}>0$.

Most monotonicity preserving schemes satisfy Assumption I. In this paper, we pay particular attention to the second order flux based MUSCL schemes. We recall some schemes for scalar conservation laws as follows:

(i) Second order upwinding flux based MUSCL scheme which works when $f^{\prime}>0$ :

$$
\begin{aligned}
& u_{j}^{n+1 / 2}=u_{j}^{n}-\lambda\left(\hat{f}_{j+1 / 2}^{n}-\hat{f}_{j-1 / 2}^{n}\right), \\
& u_{j}^{n+1}=\frac{1}{2}\left[u_{j}^{n}+u_{j}^{n+1 / 2}-\lambda\left(\hat{f}_{j+1 / 2}^{n+1 / 2}-\hat{f}_{j-1 / 2}^{n+1 / 2}\right)\right],
\end{aligned}
$$

and

$$
\hat{f}_{j+1 / 2}^{k / 2}=f\left(u_{j}^{k / 2}\right)+\frac{1}{2} m\left(f\left(u_{j+1}^{k / 2}\right)-f\left(u_{j}^{k / 2}\right), f\left(u_{j}^{k / 2}\right)-f\left(u_{j-1}^{k / 2}\right)\right),
$$

with

$$
m(a, b)= \begin{cases}a, & \text { if } a b>0 \text { and }|a| \leq|b| \\ b, & \text { if } a b>0 \text { and }|a|>|b| \\ 0, & \text { if } a b \leq 0\end{cases}
$$


(ii) The second order flux based MUSCL scheme with Lax-Friedrichs' splitting:

$$
\begin{aligned}
& u_{j}^{n+1 / 2}=u_{j}^{n}-\lambda\left(\hat{f}_{j+1 / 2}\left(u^{n}\right)-\hat{f}_{j-1 / 2}\left(u^{n}\right)\right), \\
& u_{j}^{n+1}=\frac{1}{2}\left[u_{j}^{n}+u_{j}^{n+1 / 2}-\lambda\left(\hat{f}_{j+1 / 2}\left(u^{n+1 / 2}\right)-\hat{f}_{j-1 / 2}\left(u^{n+1 / 2}\right)\right)\right],
\end{aligned}
$$

where

$$
\begin{gathered}
\hat{f}_{j+1 / 2}=\hat{f}_{j+1 / 2}^{-}+\hat{f}_{j+1 / 2}^{+}, \\
\hat{f}_{j+1 / 2}^{+}=f^{+}\left(u_{j}\right)+\frac{1}{2} m\left(f^{+}\left(u_{j+1}\right)-f^{+}\left(u_{j}\right), f^{+}\left(u_{j}\right)-f^{+}\left(u_{j-1}\right)\right),
\end{gathered}
$$

with

$$
m(a, b)= \begin{cases}a, & \text { if } a b>0 \text { and }|a| \leq|b| \\ b, & \text { if } a b>0 \text { and }|a|>|b| \\ 0, & \text { if } a b \leq 0\end{cases}
$$

and

$$
\hat{f}_{j+1 / 2}^{-}=f^{-}\left(u_{j+1}\right)-\frac{1}{2} m\left(f^{-}\left(u_{j+1}\right)-f^{-}\left(u_{j}\right), f^{-}\left(u_{j+2}\right)-f^{-}\left(u_{j+1}\right)\right),
$$

where

$$
f^{-}(u):=\frac{1}{2}(f(u)-a u), \quad f^{+}(u):=\frac{1}{2}(f(u)+a u)
$$

with $a>\max \left|f^{\prime}(u)\right|$. MUSCL scheme (1.4) is TVD and hence monotonicity preserving.

(iii) Godunov scheme: The flux function of the Godunov scheme is

$$
\bar{f}_{j+1 / 2}^{n}=\left\{\begin{array}{ll}
\min _{u_{j}^{n} \leq u \leq u_{j+1}^{n}} f(u), & \text { if } u_{j}^{n} \leq u_{j+1}^{n}, \\
\max _{u_{j}^{n} \geq u \geq u_{J+1}^{n}} f(u), & \text { if } u_{j}^{n} \geq u_{j+1}^{n}
\end{array} .\right.
$$

The Godunov scheme is $l^{1}$-contracting and hence monotonicity preserving.

All schemes listed in (i)-(iii) have Lipschitz continuous flux functions $\bar{f}$ and satisfy Assumption I.

We intend to establish the existence of discrete shock profiles of monotonicity preserving schemes (1.2) with end states

$$
u_{j} \rightarrow u_{ \pm} \quad \text { as } j \rightarrow \pm \infty, \quad u_{-}>u_{+} .
$$

The speed of the shock profile is

$$
s=\frac{f\left(u_{+}\right)-f\left(u_{-}\right)}{u_{+}-u_{-}} .
$$

We know that for a traveling wave of the scalar equation (1.1) to exist, it is necessary that the chord condition

$$
\frac{f(u)-f\left(u_{-}\right)}{u-u_{-}}<\frac{f\left(u_{+}\right)-f\left(u_{-}\right)}{u_{+}-u_{-}} \text {for all } u_{+}<u<u_{-}
$$

holds. We collect our assumptions, besides Assumption I, in the following:

Assumption II. We assume $u_{-}>u_{+}, \lambda s=l / m>0$, where $l$ and $m$ are integers, and that the chord condition (1.8) holds. 
Our results remain valid for the case $u_{-}<u_{+}$with the inequality in (1.8) reversed. For simplicity, we only present the proofs in the case given by Assumption II.

The equations of the shock profiles are

$$
\begin{gathered}
u_{j}^{k+1}=u_{j}^{k}-\lambda\left(\bar{f}_{j+1 / 2}\left(u^{k}\right)-\bar{f}_{j-1 / 2}\left(u^{k}\right)\right), \quad k=0,1,2, \ldots, m-1, \\
u_{j}^{0}=u_{j}, \quad u_{j}^{m}=u_{j-l}, \\
u_{j} \rightarrow u_{ \pm} \quad \text { as } j \rightarrow \pm \infty .
\end{gathered}
$$

To prove the existence of solutions of (1.9), we take summation on (1.9a) to obtain

$$
u_{j}^{m}=u_{j}-\lambda \sum_{k=0}^{m-1}\left(\bar{f}_{j+1 / 2}\left(u^{k}\right)-\bar{f}_{j-1 / 2}\left(u^{k}\right)\right) .
$$

We see that system (1.9) is equivalent to

$$
\begin{aligned}
& u_{j-l}=u_{j}-\lambda \sum_{k=0}^{m-1}\left(\bar{f}_{j+1 / 2}\left(u^{k}\right)-\bar{f}_{j-1 / 2}\left(u^{k}\right)\right), \\
& u_{j}^{k+1}=u_{j}^{k}-\lambda\left(\bar{f}_{j+1 / 2}\left(u^{k}\right)-\bar{f}_{j-1 / 2}\left(u^{k}\right)\right), \quad k=0,1,2, \ldots, m-1, \\
& u_{j}^{0}=u_{j}, \quad u_{j} \rightarrow u_{ \pm} \quad \text { as } j \rightarrow \pm \infty .
\end{aligned}
$$

To prove that solutions of (1.9) exist, we first modify and restrict $\left(1.9^{\prime}\right)$ as follows:

$$
\begin{aligned}
& u_{j-l}=u_{j}+\epsilon \Delta_{j-l} u-\lambda \sum_{k=0}^{m-1}\left(\bar{f}_{j+1 / 2}\left(u^{k}\right)-\bar{f}_{j-1 / 2}\left(u^{k}\right)\right), \\
& L+1 \leq j-l \leq M-1, \\
& u_{j}=u_{-} \quad \text { for } j \leq L, \quad u_{j}=u_{+} \quad \text { for } j \geq M,
\end{aligned}
$$

where $\Delta_{k} u:=u_{k+1}-2 u_{k}+u_{k-1}$ and

$$
\begin{aligned}
& u_{j}^{k+1}=u_{j}^{k}-\lambda\left(\bar{f}_{j+1 / 2}\left(u^{k}\right)-\bar{f}_{j-1 / 2}\left(u^{k}\right)\right), \quad k=0,1,2, \ldots, m-1, \\
& u_{j}^{0}=u_{j} .
\end{aligned}
$$

Our program for establishing the existence of shock profiles of (1.2) has three steps. First, we shall prove the existence of solutions of (1.10). Then we shall let $-L, M \rightarrow$ $\infty$ in (1.10) in a suitable manner so that (1.9c) holds, and then $\epsilon \rightarrow 0+$ in (1.10) to establish the existence of solutions of (1.9).

We divide this paper into three sections after this one: In Section 2, we prove the following theorem:

Theorem 1.1. There exists a decreasing solution of (1.10) for all monotonicity preserving schemes with continuous flux function $\bar{f}$.

Here, we introduce some notation. For a decreasing function $u_{j}$ with $u_{j} \rightarrow u_{ \pm}$ as $j \rightarrow \pm \infty$, we use the integer $J(u)$ to denote the location $j$ where $u_{j}$ crosses $\left(u_{-}+u_{+}\right) / 2$, that is $u_{j} \geq\left(u_{+}+u_{-}\right) / 2$ for $j \leq J(u)$ and $u_{j}<\left(u_{-}+u_{+}\right) / 2$ for $j>J(u)$. We define the function $V_{j}$ as

$$
V_{j}= \begin{cases}u_{-} & \text {if } j \leq J(u), \\ u_{+}, & \text {if } j>J(u) .\end{cases}
$$


We shall also use the notations $f_{ \pm}:=f\left(u_{ \pm}\right)$in this paper. In Sections 3 and 4 , we prove that if a monotonicity preserving scheme satisfies the following condition (1.12), then it has shock profiles. We state these results precisely as follows:

Theorem 1.2. Traveling wave equation (1.9) for a monotonicity preserving scheme with continuous flux $\bar{f}$ has a solution if any solution of (1.10) with $-L=M=N$ satisfies

$$
\sum_{k=0}^{m-1} \sum_{j=-N+1}^{N}\left|u_{j}^{k}-V_{j}^{k}\right| \leq C
$$

where $C>0$ is a constant independent of $N$ and $\epsilon>0$.

To verify the condition (1.12), we usually start with the following lemma proved in Section 4:

Lemma 1.3. Let $u_{j}$ be a decreasing solution of (1.10) with $-L=M=N>0$. Then $u_{j}$ satisfies

$$
-\sum_{j=-N}^{N-1} \sum_{k=0}^{m-1}\left[\frac{l}{m}\left(u_{-}-u_{j}^{k}\right)-\lambda\left(f_{-}-\bar{f}_{j+1 / 2}^{k}\right)\right] \leq C,
$$

where $C$ is bounded uniformly in $N$ and $\epsilon \in[0,1]$.

In Section 5, we establish the existence of discrete traveling waves for all monotonicity preserving schemes (1.2) with $C^{1}$ flux function $\bar{f}$. We also prove that the existence of discrete shock profiles for the second order flux based upwinding MUSCL scheme (1.3), the second order flux based MUSCL scheme (1.4) with LaxFriedrichs' splitting, and the Godunov scheme, in Sections 6, 7 and 8, respectively.

\section{Existence OF SOlutions of (1.10)}

In this section, we shall prove that for any monotonicity preserving scheme with continuous flux function for scalar conservation laws, the modified shock profile equation, (1.10), has a solution. To this end, we further modify (1.10) as

$$
\begin{gathered}
u_{j-l}=u_{j}+\epsilon \Delta_{j-l} u-\mu \lambda \sum_{k=0}^{m-1}\left(\bar{f}_{j+1 / 2}\left(u^{k}\right)-\bar{f}_{j-1 / 2}\left(u^{k}\right)\right), \\
L+1 \leq j-l \leq M-1, \\
u_{j}=u_{-} \text {for } j \leq L, \text { and } \quad u_{j}=u_{+} \text {for } j \geq M,
\end{gathered}
$$

where $\mu \in[0,1]$ is a constant and

$$
\begin{aligned}
& u_{j}^{k+1}=u_{j}^{k}-\mu \lambda\left(\bar{f}_{j+1 / 2}\left(u^{k}\right)-\bar{f}_{j-1 / 2}\left(u^{k}\right)\right), \quad k=0,1,2, \ldots, m-1, \\
& u_{j}^{0}=u_{j} .
\end{aligned}
$$

We note that the iteration (2.1c) is still a monotonicity preserving scheme since we can regard $\mu \lambda$ as just another smaller $\lambda$ (see Assumption I). Therefore, if $u_{j}$ is 
monotone, then $u^{1}$ determined by (2.1c) is also monotone of the same type. We introduce a new vector variable

$$
v_{j}=\left(\begin{array}{c}
v_{j}^{(1)} \\
v_{j}^{(2)} \\
\cdot \\
\cdot \\
\cdot \\
v_{j}^{(l+1)}
\end{array}\right):=\left(\begin{array}{c}
u_{j} \\
u_{j-1} \\
\cdot \\
\cdot \\
\cdot \\
u_{j-l}
\end{array}\right), \quad \text { i.e., } v_{j}^{(k+1)}=u_{j-k}
$$

with which we can rewrite (2.1a) into a system of first order difference equations

$$
\begin{aligned}
& v_{j}^{(1)}=v_{j-1}^{(l)}-\epsilon\left(v_{j-1}^{(l-1)}-2 v_{j-1}^{(l)}+v_{j-1}^{(l+1)}\right)-\mu g(j, u, m), \\
& v_{j}^{(2)}=v_{j-1}^{(1)}, \\
& v_{j}^{(3)}=v_{j-1}^{(2)} \\
& \cdots \\
& v_{j}^{(l+1)}=v_{j-1}^{(l)} \\
& L+l+1 \leq j \leq M+l-1 .
\end{aligned}
$$

The matrix form for $(2.2)$ is

$$
v_{j}=A v_{j-1}+\mu F(j, u, \mu, L, M),
$$

where the constant $(l+1) \times(l+1)$ matrix $A$ is defined as such that $v_{j}=A v_{j-1}$ is (2.2) when $\mu=0$, i.e.,

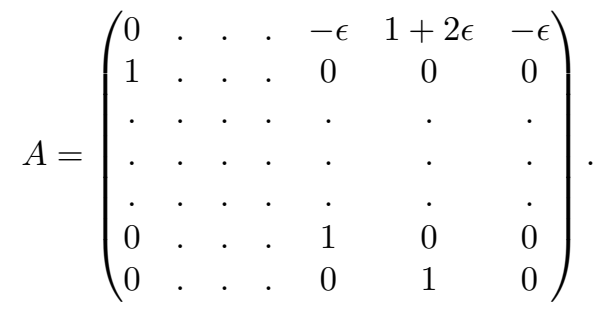

The boundary condition (2.1b) becomes

$$
v_{j}^{(k+1)}= \begin{cases}u_{+}, & \text {if } j-k \geq M, \\ u_{-}, & \text {if } j-k \leq L .\end{cases}
$$

We see that systems (2.1) and (2.3) are equivalent. The system

$$
v_{j}=A v_{j-1}
$$

has general solutions of the form $Y_{j} C$ where $Y_{j}=A^{j-(L+l+1)}$ is the Wronskian matrix of (2.4) and $C$ is a constant vector in $\mathbb{R}^{l+1}$.

Lemma 2.1. The boundary value problem

$$
\begin{aligned}
& v_{j}=A v_{j-1}, \\
& v_{j}^{(k+1)}= \begin{cases}u_{+}, & \text {if } j-k \geq M, \\
u_{-}, & \text {if } j-k \leq L,\end{cases}
\end{aligned}
$$


has a unique solution. Moreover, this solution, denoted by $w$, is strictly decreasing in the sense that $v_{j}^{(k)}>v_{j}^{(k+1)}$ for $j=L+l+1, \ldots, M+l-2, k=1,2, \ldots, l$ and $L+1 \leq j-k \leq M$.

Proof. Problem (2.5) is equivalent to the following boundary value problem:

$$
\begin{aligned}
& 0=u_{j}-u_{j-l}+\epsilon \Delta u_{j-l}, \quad L+l+1 \leq j \leq M+l-1, \\
& u_{M}=u_{M+1}=\cdots=u_{M+l-1}=u_{+}, \quad u_{L}=u_{-} .
\end{aligned}
$$

To prove the existence of solutions of (2.5), we consider the initial value problem

$$
\begin{aligned}
& 0=u_{j}-u_{j-l}+\epsilon \Delta u_{j-l}, \quad L+l+1 \leq j \leq M+l-1, \\
& u_{M}=u_{M+1}=\cdots=u_{M+l-1}=u_{+}, \quad u_{M-1}=b .
\end{aligned}
$$

The solution of the initial value problem (2.6) is unique and depends on initial data and hence $u_{+}$and $b$ continuously. We claim that the solution of (2.6) is monotone. To this end, we assume that $b \geq u_{+}$. The case where $b<u_{+}$can be handled similarly. Then from (2.6) we have

$$
u_{M-1} \geq u_{M} \geq u_{M+1} \geq \cdots \geq u_{M+l-1} .
$$

Assume, for induction, that

$$
u_{j-l} \geq u_{j-l+1} \geq \cdots \geq u_{j}
$$

which holds at least for $j=M+l-1$. Then $(2.6)_{1}$ leads to

$$
\epsilon\left(u_{j-l-1}-u_{j-l}\right)=\epsilon\left(u_{j-l}-u_{j-l+1}\right)+\left(u_{j-l}-u_{j}\right) \geq 0,
$$

and hence (2.7) holds for $j=j-1$. By induction, the claim is proved. If $b=u_{+}$, then the solution of (2.6) is the constant $u_{+}$. If $b=u_{-}>u_{+}$, by the monotonicity of the solution $u_{j}$ of $(2.6)$, we have $u_{L}>u_{-}$. Since $u_{L}$ depends on $b$ continuously, there is a point $b \in\left(u_{+}, u_{-}\right)$such that $u_{L}=u_{-}$. Thus there is a solution of $(2.5)$.

To prove the uniqueness of the solution of $(2.5)$, it suffices to prove that $u_{L}$ is a strictly monotone function of $b$. To this end, we let $u_{j}$ and $\bar{u}_{j}$ denote solutions of (2.5) with $u_{M-1}=b$ and $\bar{u}_{M-1}=\bar{b}$, respectively. Without loss of generality, we assume $\bar{b}>b$. Then the estimate

$$
\bar{u}_{j-l}-u_{j-l} \geq \bar{u}_{j-l+1}-u_{j-l+1} \geq \cdots \geq \bar{u}_{j}-u_{j}
$$

holds for $j=M+l-1$. Assume that (2.8) holds for $j \leq M+l-1$, then equation (2.6) implies

$$
\begin{aligned}
& \epsilon\left[\left(\bar{u}_{j-l-1}-u_{j-l-1}\right)-\left(\bar{u}_{j-l}-u_{j-l}\right)\right]=\epsilon\left[\left(\bar{u}_{j-l}-u_{j-l}\right)-\left(\bar{u}_{j-l+1}-u_{j-l+1}\right)\right] \\
& \quad+\left[\left(\bar{u}_{j-l}-u_{j-l}\right)-\left(\bar{u}_{j}-u_{j}\right)\right] \geq 0 .
\end{aligned}
$$

Thus, inequalities (2.8) hold for $j=j-1$. By induction, inequality (2.8) holds for any $L+l \leq j \leq M+l-1$. Then estimates (2.8) yield the desired strict monotonicity of $u_{L}, \bar{u}_{L}-u_{L} \geq \bar{u}_{M-1}-u_{M-1}=\bar{b}-b>0$, which implies the uniqueness of the solution of $(2.5)$.

Lemma 2.2. Let $\bar{Y}_{M+l-1}$ denote the first $l$ rows of the Wronskian $Y_{M+l-1}$ of (2.4) at $j=M+l-1$, and $\bar{y}_{l+1, L+l}$ is the $l+1$-th row of $Y_{L+l}$. Then the matrix

$$
\left(\begin{array}{l}
\bar{Y}_{M+l-1} \\
\bar{y}_{l+1, L+l}
\end{array}\right)
$$

is invertible. 
Proof. Since the Wronskian matrix $Y_{j}$ is invertible, the rank of $\bar{Y}_{M+l-1}$ is $l$. Assume, for contradiction, that the matrix (2.9) is not invertible. Then the last row must be a linear combination of the other $l$ rows of $\bar{Y}_{M+l-1}$, that is, there are constants $a_{1}, a_{2}, \ldots, a_{l}$ such that

$$
\bar{y}_{l+1, L+l}=a_{1} \bar{y}_{1, M+l-1}+a_{2} \bar{y}_{2, M+l-1}+\cdots+a_{l} \bar{y}_{l, M+l-1},
$$

where $\bar{y}_{k, M+l-1}$ is the $k$-th row of $Y_{M+l-1}$. Since the general solution of (2.4) is $Y_{j} C$, the solution of boundary value problem of (2.5) satisfies

$$
\left(\begin{array}{c}
\bar{Y}_{M+l-1} \\
\bar{y}_{l+1, L+l}
\end{array}\right) C=\left(\begin{array}{c}
u_{+} \\
\cdot \\
\cdot \\
\cdot \\
u_{+} \\
u_{-}
\end{array}\right)
$$

for some vector $C \in \mathbb{R}^{l+1}$. After some row manipulations in (2.11) by using (2.10), we have

$$
\left(\begin{array}{c}
\bar{Y}_{M+l-1} \\
0
\end{array}\right) C=\left(\begin{array}{c}
u_{+} \\
\cdot \\
\cdot \\
\cdot \\
u_{+} \\
u_{-}-u_{+}\left(a_{1}+a_{2}+\cdots+a_{l}\right)
\end{array}\right) .
$$

We note that in (2.12), $a_{1}, a_{2}, \ldots, a_{l}$ depends only on $\bar{Y}_{M+l-1}$ and $\bar{y}_{l+1, L+l}$ which are independent of $u_{+}$and $u_{-}$. Thus, (2.12) cannot hold for arbitrary $u_{-}$and $u_{+}$which means that $(2.5)$ does not have solution for some $u_{ \pm}$. This contradicts Lemma 2.2. This contradiction proves our assertion.

Now, we use the technique of variation of constants to rewrite the problem (2.3) as follows. Assume the solution of (2.3) has the form $v_{j}=Y_{j} C_{j}$. Plugging this form into (2.3), we get

$$
C_{j}-C_{j-1}=\mu Y_{j}^{-1} F(j, v),
$$

which leads to

$$
C_{j}=C_{M+l-1}-\sum_{i=j+1}^{M+l-1} \mu Y_{i}^{-1} F(i, v)
$$

and hence

$$
v_{j}=Y_{j} C-\sum_{i=j+1}^{M+l-1} \mu Y_{j} Y_{i}^{-1} F(i, v) .
$$

The boundary condition (2.3b) determines the constant vector $C$ in (2.15):

$$
C=C(v, \mu)=\left(\bar{Y}_{M+l-1} \bar{y}_{l+1, L+l}\right)^{-1}\left(\begin{array}{c}
u_{+} \\
\cdot \\
\cdot \\
\cdot \\
u_{+} \\
u_{-}+B(v, \mu)
\end{array}\right) \text {, }
$$


where

$$
B(v, \mu, L, M):=\sum_{i=j+1}^{M+l-1} \mu\left[\begin{array}{lllll}
0 & \cdots & 0 & 1
\end{array}\right] Y_{L+l} Y_{i}^{-1} F(i, v) .
$$

We define the operator $T: \mathbb{R}^{(M-L+1) \times(l+1)} \times[0,1] \rightarrow \mathbb{R}^{(M-L+1) \times(l+1)}$ by

$$
(\mathbf{T}(v, \mu))_{j}:=Y_{j} C(\bar{v}, \mu)-\sum_{i=j+1}^{M+l-1} \mu Y_{j} Y_{i}^{-1} F(i, \bar{v}),
$$

where the notation $\bar{v}$ is defined as

$$
\bar{v}_{j}^{(k+1)}= \begin{cases}u_{+}, & \text {if } j-k \geq M, \\ u_{-}, & \text {if } j-k \leq L, \\ v_{j}^{(k+1)}, & \text { else, }\end{cases}
$$

to enforce the boundary conditions. The choice of $C(\bar{v}, \mu)$ made in (2.16) guarantees that $(\mathbf{T}(v, \mu))_{j}$ satisfies the boundary condition (2.3c). A straightforward calculation verifies that the boundary value problem (2.3) is equivalent to the fixed point problem $v=\mathbf{T}(v, \mu)$. To prove the existence of solutions of (1.10), it suffices to prove that there is a fixed point of $\mathbf{T}(., 1)$.

Theorem 2.3. If the numerical flux function $\bar{f}_{j+1 / 2}($.$) of a monotone preserving$ scheme is continuous in its variables, then the problem (1.10) has a strictly decreasing solution.

Proof. Since the function $\bar{f}_{j+1 / 2}(v)$ and hence $F(j, v)$ is continuous in $v$ for all $j$, the operator $\mathbf{T}: \mathbb{R}^{(M-L+1) \times(l+1)} \times[0,1] \rightarrow \mathbb{R}^{(M-L+1) \times(l+1)}$ is also continuous. Furthermore, because the range of $\mathbf{T}$ is of finite dimension, the operator $\mathbf{T}$ is compact. Now, we recall a fixed point theorem of Leray-Shauder type as follows:

Lemma 2.4 (Ma $)$. Let $X$ be a real normed vector space and $\Omega$ a bounded open subset of $X$. Let $T: \bar{\Omega} \times[0,1] \rightarrow X$ be a compact operator. If

(i) $\mathbf{T}(x, \mu) \neq x$ for $x \in \partial \Omega, \mu \in[0,1]$, and

(ii) the Leray-Shauder degree $D_{I}(\mathbf{T}(., 0)-I, \Omega) \neq 0$,

then $T(x, 1)=x$ has at least one solution in $\Omega$.

We choose $X$ in the lemma above as

$$
X=\left\{v \in \mathbb{R}^{(M-L+1) \times(l+1)}: v \text { satisfy }(2.3 b)\right\}
$$

and the open subset $\Omega$ of $X$ as

$$
\Omega:=\left\{v \in X: v_{j}^{(k)}>v_{j}^{(k+1)} \text { for } L \leq j-k \leq M, k=1,2, \ldots, l\right\} .
$$

Since $\mathbf{T}(v, \mu)$ satisfies $(2.3 \mathrm{~b})$ for any $v \in \mathbb{R}^{(M-L+1) \times(l+1)}$, $\mathbf{T}$ is a compact operator from $\bar{\Omega}$ to $X$. We observe that

$$
\mathbf{T}(v, 0)=w \in \Omega,
$$

where $w$ is the solution of (2.1) when $\mu=0$, provided by Lemma 2.1, which is independent of $v$. This implies that

$$
D_{I}(\mathbf{T}(., 0)-I, \Omega)=1 .
$$

Thus, condition (ii) of Lemma 2.4 is satisfied. To verify condition (i) of Lemma 2.4, we assume its contrary, i.e., there is a solution $v$ of $\mathbf{T}(v, \mu)=v$ for some $v \in \partial \Omega$ 
and $\mu \in(0,1]$. Since $v \in \partial \Omega$, it satisfies $v_{j}^{(k)} \geq v_{j}^{(k+1)}$ for all $L \leq j-k \leq M$ and $k=1,2, \ldots, l+1$ with " $="$ holds for some $(j, k)=\left(j_{0}, k_{0}\right), L \leq j_{0}-k_{0} \leq M$, $k_{0}=1,2, \ldots, l+1$. Then there is a solution $u$ of $(2.1)$, with $v_{j}^{(k+1)}=u_{j-k}$, for some $\mu \in(0,1)$ which satisfies $u_{j} \geq u_{j+1}$ and $u_{j_{0}}=u_{j_{0}+1}$ for some $L \leq j_{0}<M$. We claim this will lead to a contradiction. To this end, we further select $j_{0}$ and $j_{1}>j_{0}$ such that

$$
u_{i} \geq u_{j_{0}}=u_{j_{0}+1}=\cdots=u_{j_{1}} \geq u_{j}
$$

for $i<j_{0}$ and $j>j_{1}$, where strict inequality

$$
u_{i}>u_{j_{0}}
$$

holds when $L<j_{0}$, and

$$
u_{j_{1}}>u_{j}
$$

holds when $j_{1}<M$. We see that at least one of (2.22) and (2.23) is true by the boundary condition (2.3b). By Assumption I, the iteration defined by $(2.1 \mathrm{c})$ is also monotonicity preserving since we can treat $\mu \lambda$ as a new and smaller $\lambda$ in (1.2). Since $u_{j}$ is decreasing, the m-th iteration, defined in (2.1c) of $u$ is also decreasing and hence

$$
u_{j_{0}+l}^{m} \geq u_{j_{1}+l}^{m}
$$

Then equations (2.1) and (2.21)-(2.23) yield

$$
0>-\epsilon \Delta_{j_{0}} u+\epsilon \Delta_{j_{1}} u=u_{j_{0}+l}^{m}-u_{j_{1}+l}^{m} \geq 0,
$$

which is a contradiction. Thus both conditions of Lemma 2.4 are met and hence there is a solution of $v=T(v, 1)$ which is a solution of $(1.10)$.

\section{The EXIstence of SHOCK PROfiles}

In this section, we shall prove the existence of shock profiles for a class of monotonicity preserving schemes by passing the limit $L \rightarrow-\infty, M \rightarrow \infty$ in a suitable manner and then $\epsilon \rightarrow 0+$ in (1.10). For convenience, we first take $-L=M=N>0$.

Lemma 3.1. Let $u_{j},|j| \leq N$ be a solution of (1.10) with $-L=M=N>0$. Then $u_{j}$ satisfies

$$
\begin{aligned}
& \epsilon\left(u_{+}-u_{-}\right)=\epsilon\left(u_{+}-u_{N-1}\right)+\sum_{j=-N+1}^{-N+l-1}(j+N-l)\left(u_{+}-u_{j}\right) \\
& +\sum_{k=0}^{m-1} \sum_{j=-N+1}^{N}\left[\frac{l}{m}\left(u_{+}-u_{j}\right)-\lambda\left(\bar{f}_{N+l-1 / 2}^{k}-\bar{f}_{j+l-1 / 2}^{k}\right)\right],
\end{aligned}
$$

where $\bar{f}_{j+1 / 2}^{k}:=\bar{f}_{j+1 / 2}\left(u^{k}\right)$. 
Proof. Taking $\sum_{J=-N+l+1}^{N+l} \sum_{j=J}^{N+l-1}$ on (1.10), we obtain

$$
\begin{aligned}
- & \sum_{J=i+l+1}^{N+l} \sum_{j=J}^{N+l-1} \epsilon \Delta_{j-l} u \\
= & \epsilon\left(u_{+}-u_{-}\right)-2 N \epsilon\left(u_{+}-u_{N-1}\right) \\
= & \sum_{J=-N+l+1}^{N+l} \sum_{j=J}^{N+l-1}\left[u_{j}-u_{j-l}-\lambda \sum_{k=0}^{m-1}\left(\bar{f}_{j+1 / 2}^{k}-\bar{f}_{j-1 / 2}^{k}\right)\right] \\
= & \sum_{J=-N+1}^{N}\left[\sum_{j=J}^{J+l-1}\left(u_{+}-u_{j}\right)-\lambda \sum_{k=0}^{m-1}\left(\bar{f}_{N+l-1 / 2}^{k}-\bar{f}_{J+l-1 / 2}^{k}\right)\right] .
\end{aligned}
$$

$$
\begin{aligned}
& \sum_{J=-N+1}^{N} \sum_{j=J}^{J+l-1}\left(u_{+}-u_{j}\right)=\sum_{j=-N+1}^{-N+l-1} \sum_{J=-N+1}^{j}\left(u_{+}-u_{j}\right) \\
+ & \sum_{j=-N+l}^{N} \sum_{J=j-l+1}^{j}\left(u_{+}-u_{j}\right)+\sum_{j=N}^{N+l-2} \sum_{J=j-l+1}^{N}\left(u_{+}-u_{j}\right) \\
= & \sum_{j=-N+1}^{-N+l-1}(j+N)\left(u_{+}-u_{j}\right)+\sum_{j=-N+l}^{N} l\left(u_{+}-u_{j}\right) \\
= & \sum_{j=-N+1}^{N+l-1}(j+N-l)\left(u_{+}-u_{j}\right)+\sum_{j=-N+1}^{N} l\left(u_{+}-u_{j}\right) .
\end{aligned}
$$

Plugging (3.3) into (3.2), we obtain (3.1).

Lemma 3.2. Solutions of (1.10) with $-L=M=N$ satisfies

$$
\begin{aligned}
& \epsilon\left(u_{N-1}-u_{+}\right)+\lambda \sum_{k=0}^{m-1}\left[\bar{f}_{N+l-1 / 2}^{k}-f_{+}\right] \\
& =\epsilon\left(u_{-}-u_{-N+1}\right)+\sum_{j=-N+1}^{-N+l}\left(u_{-}-u_{j}^{m}\right)+\lambda \sum_{k=0}^{m-1}\left[\bar{f}_{-N+1 / 2}^{k}-f_{-}\right] .
\end{aligned}
$$

Proof. By taking $\sum_{j=-N+l+1}^{N+l-1}$ on (1.10), we obtain

$$
\begin{aligned}
& \epsilon\left(u_{N-1}-u_{+}\right)+\lambda \sum_{k=0}^{m-1}\left[\bar{f}_{N+l-1 / 2}^{k}-f_{+}\right] \\
& =\epsilon\left(u_{-}-u_{-N+1}\right)+\sum_{j=-N+1}^{-N+l}\left(u_{+}-u_{j}\right)+\lambda \sum_{k=0}^{m-1}\left[\bar{f}_{-N+l+1 / 2}^{k}-f_{+}\right] .
\end{aligned}
$$


We also have

$$
\begin{aligned}
& \sum_{j=-N+1}^{-N+l}\left(u_{-}-u_{j}\right)+\lambda \sum_{k=0}^{m-1}\left[\bar{f}_{-N+l+1 / 2}^{k}-f_{-}\right] \\
= & \sum_{j=-N+1}^{-N+l-1}\left(u_{-}-u_{j}\right)+\lambda \sum_{k=0}^{m-1}\left[\bar{f}_{-N+l-1+1 / 2}^{k}-f_{-}\right] \\
+ & \left(u_{-}-u_{-N+l}\right)+\lambda \sum_{k=0}^{m-1}\left[\bar{f}_{-N+l+1 / 2}^{k}-\bar{f}_{-N+l-1+1 / 2}^{k}\right] \\
= & \sum_{j=-N+1}^{-N+l-1}\left(u_{-}-u_{j}\right)+\lambda \sum_{k=0}^{m-1}\left[\bar{f}_{-N+l-1+1 / 2}^{k}-f_{-}\right]+\left(u_{-}-u_{-N+l}^{m}\right) \\
= & \cdots=\sum_{j=-N+1}^{-N+l}\left(u_{-}-u_{j}^{m}\right)+\lambda \sum_{k=0}^{m-1}\left[\bar{f}_{-N+1 / 2}^{k}-f_{-}\right] .
\end{aligned}
$$

Plugging (3.6) into (3.5) and using Rankine-Hugoniot condition for shocks, we conclude (3.4).

Lemma 3.3. Let $u_{j}$ be a decreasing function satisfying (1.10). Then

(i) if $u_{j}=u_{-}$for some $j_{0} \geq-N+1$, then $u_{j}=u_{-}$for $-N+1 \leq j \leq N-1$;

(ii) if $u_{j}=u_{+}$for some $j_{0} \leq N-1$, then $u_{j}=u_{+}$for $-N+1 \leq j \leq N-1$.

Proof. We see that $u_{j}=u_{-}$holds for $j \leq j_{0}$. Assume, for induction, that $u_{j}=u_{-}$ holds for $j \leq k$. We consider (1.10a) with $j-l=k$

$$
u_{k}-u_{k+l}^{m}=\epsilon\left(u_{k+1}-2 u_{k}+u_{k-1}\right) \text {, }
$$

which implies

$$
u_{-}-u_{k+l}^{m}=\epsilon\left(u_{k+1}-u_{-}\right) \leq 0 .
$$

Since $u_{j}$, with (1.10b), is decreasing and the scheme is monotonicity preserving, we have $u_{k+l}^{m} \leq u_{-}$. This together with (3.7) yields that $u_{k+1}=u_{-}$, i.e., $u_{j}=u_{-}$holds for $j \leq k+1$. The induction is complete. The proof of assertion (ii) is similar.

Theorem 3.4. The traveling wave equation (1.9) for a monotonicity preserving scheme with continuous flux $\bar{f}$ has a solution if any decreasing solution of (1.10) with $-L=M=N$ satisfies

$$
\sum_{k=0}^{m-1} \sum_{j=-N+1}^{N}\left|u_{j}^{k}-V_{j}^{k}\right| \leq C,
$$

where $V_{j}$ is defined in (1.11) and $C>0$ is a constant bounded uniformly in $N$ and $\epsilon>0$ and

$$
\lambda \sum_{k=0}^{m-1}\left[\bar{f}_{N+l-1 / 2}^{k}-f_{+}\right] \geq 0
$$

and

$$
\sum_{j=-N+1}^{-N+l}\left(u_{-}-u_{j}^{m}\right)+\lambda \sum_{k=0}^{m-1}\left[\bar{f}_{-N+1 / 2}^{k}-f_{-}\right] \geq 0 .
$$


Proof. Let $u_{j}$ be a solution of (1.10) with $-L=M=N$ and define $L(N):=$ $-N-J(u)$ and $M(N):=N-J(u)$. Then $\bar{u}_{j}(N):=u_{j+J(u)}$ is a solution of (1.10) with $L=L(N)$ and $M=M(N)$. It is clear that $J(\bar{u})=0$ and hence $\bar{u}_{j}(N) \geq\left(u_{-}+u_{+}\right) / 2$ for $j \leq 0$ and $\bar{u}_{j}(N)<\left(u_{-}+u_{+}\right) / 2$ for $j>0$. From condition (3.8a), we have

$$
\sum_{j=L(N)+1}^{M(N)}\left|\bar{u}_{j}(N)-V_{j}\right| \leq C .
$$

We claim that $L(N) \rightarrow-\infty$ and $M(N) \rightarrow \infty$ as $N \rightarrow \infty$. Indeed, if otherwise, either $\left|L\left(N_{n}\right)\right| \leq A$ or $\left|M\left(N_{n}\right)\right| \leq A$ holds for a subsequence $\left\{N_{n}\right\}$ of $\{N\}$ and a constant $A>0$ independent of $N_{n}$. We consider the case where $\left|L\left(N_{n}\right)\right| \leq A$. The other case can be handled similarly. Since $M\left(N_{n}\right)+L\left(N_{n}\right)=2 N$, the boundedness of $L\left(N_{n}\right)$ implies that $M\left(N_{n}\right) \rightarrow \infty$ as $n \rightarrow \infty$. Since $\bar{u}_{j}\left(N_{n}\right)$ and $L\left(N_{n}\right)$ are bounded independent of $N_{n}$, there is a subsequence of $\left\{N_{n}\right\}$, denoted by $\left\{N_{n}\right\}$ again, such that $L\left(N_{n}\right)=$ a constant $L$ for large $n$ and

$$
\bar{u}_{j}\left(N_{n}\right) \rightarrow \bar{u}_{j}, \quad \text { for } L \leq j<\infty
$$

as $n \rightarrow \infty$. Using (3.9), (3.10) and that (3.9) is a nonnegative term sum, we obtain

$$
\sum_{j=L}^{\infty}\left|\bar{u}_{j}-V_{j}\right| \leq C,
$$

and hence $\bar{u}_{j} \rightarrow u_{+}$as $j \rightarrow \infty$. Applying Lemma 3.2 to $u_{j}\left(N_{n}\right)$, we have

$$
\begin{aligned}
& \epsilon\left(u_{M\left(N_{n}\right)-1}-u_{+}\right)+\lambda \sum_{k=0}^{m-1}\left[\bar{f}_{M\left(N_{n}\right)+l-1 / 2}^{k}-f_{+}\right] \\
& =\epsilon\left(u_{-}-u_{L\left(N_{n}\right)+1}\right)+\sum_{j=L\left(M_{n}\right)+1}^{L\left(N_{n}\right)+l}\left(u_{-}-u_{j}^{m}\right)+\sum_{k=0}^{m-1}\left[\bar{f}_{L\left(N_{n}\right)+1 / 2}^{k}-f_{-}\right],
\end{aligned}
$$

where we omitted $N$ from $u_{j}(N)$. Due to the fact that $\bar{u}_{j} \rightarrow u_{+}$as $j \rightarrow \infty$ and that $\bar{u}_{j}$ is monotone, the left hand side of (3.12) tends to zero as $n \rightarrow \infty$. Applying $(3.8 \mathrm{~b})$ and (3.8c) to (3.12) and letting $n \rightarrow \infty$, we have

$$
0 \geq \epsilon \lim _{n \rightarrow \infty}\left(u_{-}-u_{L\left(N_{n}\right)+1}(N)\right)=\epsilon\left(u_{-}-\bar{u}_{L+1}\right)
$$

and hence $\bar{u}_{L+1}=u_{-}$. Note that $\bar{u}_{j}$ satisfies (1.10a) for $L+1 \leq j-l<\infty$ with $u_{+} \leq$ $\bar{u}_{j} \leq u_{-}$. Then Lemma 3.6 states that $\bar{u}_{j}=u_{-}$for all $j \geq L$ which is contradictory to (3.11). This contradiction proves that $L(N) \rightarrow-\infty$ and $M(N) \rightarrow \infty$ as $N \rightarrow \infty$. Thus, $\bar{u}_{j}$ is defined for $-\infty<j<\infty$ and satisfies (1.10) with $-L=M=\infty$. 
It is obvious that $\bar{u}_{j}$ depends on $\epsilon>0$ and we denote this dependence by $\bar{u}_{j}(\epsilon)$. Since $u_{+} \leq \bar{u}_{j}(\epsilon) \leq u_{-}$, there is a sequence $\left\{\epsilon_{n}\right\}$ such that $\epsilon_{n} \rightarrow 0+$ and

$$
\bar{u}_{j}\left(\epsilon_{n}\right) \rightarrow \bar{u}_{j}, \quad \text { for all } j
$$

as $n \rightarrow \infty$. It is clear from the continuity of (1.10) that the limit $\bar{u}_{j}$ satisfies (1.9a) and (1.9b). From (3.11), we see that $\bar{u}_{j}$ also satisfies (1.9c).

Theorem 3.5. The traveling wave equation (1.9) for a monotonicity preserving scheme with continuous flux $\bar{f}$ has a solution if any solution of (1.10) with $-L=M=N$ satisfies

$$
\sum_{k=0}^{m-1} \sum_{j=-N+1}^{N}\left|u_{j}^{k}-V_{j}^{k}\right| \leq C,
$$

where $C>0$ is a constant bounded uniformly in $N$ and $\epsilon>0$.

Proof. By Theorem 3.4, it suffices to prove that any decreasing solution $u_{j}$ of (1.9) satisfies (3.8b) and (3.8c). To this end, we consider the identity

$$
\begin{aligned}
\lambda \sum_{k=0}^{m-1}[ & \left.\bar{f}_{N+l-1 / 2}^{k}-f_{+}\right] \\
= & \left(u_{N+l}-u_{+}\right)-\lambda \sum_{k=0}^{m-1}\left[\bar{f}_{N+l+1-1 / 2}^{k}-f_{N+l-1 / 2}\right] \\
& +\lambda \sum_{k=0}^{m-1}\left[\bar{f}_{N+l+1-1 / 2}^{k}-f_{+}\right] \\
= & \left(u_{N+l}^{m}-u_{+}\right)+\lambda \sum_{k=0}^{m-1}\left[\bar{f}_{N+l+1-1 / 2}^{k}-f_{+}\right] \\
= & \cdots \\
= & \sum_{j=N+l}^{N+m p}\left(u_{j}^{m}-u_{+}\right)+\lambda \sum_{k=0}^{m-1}\left[\bar{f}_{N+m p+1 / 2}^{k}-f_{+}\right] .
\end{aligned}
$$

Since the scheme is a $p+q+1$-point scheme and $u_{j}=u_{+}$for $j \geq N$, we have $f_{N+m p+1 / 2}^{k}=f_{+}$for $k=0,1,2, \ldots, m-1$ and hence

$$
\lambda \sum_{k=0}^{m-1}\left[\bar{f}_{N+l-1 / 2}^{k}-f_{+}\right]=\sum_{j=N+l}^{N+m p}\left(u_{j}^{m}-u_{+}\right) \geq 0,
$$

where we used the monotonicity preserving property of the scheme.

The proof of (3.8c) is similar.

\section{The Verification of CONDition (3.14)}

To verify conditions (3.14) for various schemes, we need the following preparations. 
Lemma 4.1. Let $u_{j}$ be a solution of (1.10) with $-L=M=N>0$. Then $u_{j}$ satisfies

$$
\begin{aligned}
& \epsilon\left(u_{+}-u_{-}\right)-\sum_{j=-N+1}^{-N+l-1}(j+N-1-l)\left(u_{-}-u_{j}\right) \\
& -\frac{l(l+1)}{2}\left(u_{-}-u_{+}\right)+\lambda \sum_{k=0}^{m-1} \sum_{j=0}^{l-1}\left(\bar{f}_{-N+j+1 / 2}^{k}-\bar{f}_{N+j+1 / 2}^{k}\right) \\
& +\frac{\lambda l}{m} \sum_{k=0}^{m-1} \sum_{i=0}^{k-1}\left(\bar{f}_{N+1 / 2}^{i}-\bar{f}_{-N+1 / 2}^{i}\right) \\
& =2 N \epsilon\left(u_{+}-u_{N-1}\right)+2 N \sum_{j=-m q}^{l}\left(u_{-N+j}^{m}-u_{-}\right) \\
& +\sum_{j=-N}^{N-1} \sum_{k=0}^{m-1}\left[\frac{l}{m}\left(u_{-}-u_{j}^{k}\right)-\lambda\left(f_{-}-\bar{f}_{j+1 / 2}^{k}\right)\right] .
\end{aligned}
$$

Proof. We take $\sum_{J=-N}^{N-1} \sum_{j=-N+l+1}^{J+l}$ on (1.10) to obtain

$$
\begin{aligned}
& \epsilon\left(u_{+}-u_{-}\right)=2 N \epsilon\left(u_{+}-u_{N-1}\right) \\
& +\sum_{J=-N}^{N-1}\left[\sum_{j=1}^{l}\left(u_{-N+j}-u_{J+j}\right)-\lambda \sum_{k=0}^{m-1}\left(\bar{f}_{-N+l+1 / 2}^{k}-\bar{f}_{J+l+1 / 2}^{k}\right)\right] \\
& =2 N \epsilon\left(u_{+}-u_{N-1}\right)+\sum_{J=-N}^{N-1}\left[\sum_{j=1}^{l}\left(u_{-}-u_{J+j}\right)-\lambda \sum_{k=0}^{m-1}\left(f_{-}-\bar{f}_{J+l+1 / 2}^{k}\right)\right] \\
& +\sum_{J=-N}^{N-1}\left[\sum_{j=1}^{l}\left(u_{-N+j}-u_{-}\right)-\lambda \sum_{k=0}^{m-1}\left(\bar{f}_{-N+l+1 / 2}^{k}-f_{-}\right)\right] .
\end{aligned}
$$

The last line of (4.2) can be rewritten as

$$
\begin{aligned}
& 2 N\left[\sum_{j=1}^{l-1}\left(u_{-N+j}-u_{-}\right)-\lambda \sum_{k=0}^{m-1}\left(\bar{f}_{-N+l-1+1 / 2}^{k}-f_{-}\right)\right] \\
& +2 N\left[\left(u_{-N+l}-u_{-}\right)-\lambda \sum_{k=0}^{m-1}\left(\bar{f}_{-N+l+1 / 2}^{k}-\bar{f}_{-N+l-1+1 / 2}^{k}\right)\right] \\
& =2 N\left[\sum_{j=1}^{l-1}\left(u_{-N+j}-u_{-}\right)-\lambda \sum_{k=0}^{m-1}\left(\bar{f}_{-N+l-1+1 / 2}^{k}-f_{-}\right)\right] \\
& +2 N\left(u_{-N+l}^{m}-u_{-}\right)=\cdots \\
& =2 N \sum_{j=-m q}^{l}\left(u_{-N+j}^{m}-u_{-}\right),
\end{aligned}
$$


where we used (1.10c). By rearranging the terms in the following summation, we can see that

$$
\begin{aligned}
& \sum_{J=-N}^{N-1} \sum_{j=J+1}^{J+l}\left(u_{-}-u_{j}\right)=\sum_{j=-N+1}^{-N+l-1} \sum_{J=-N}^{j-1}\left(u_{-}-u_{j}\right) \\
& +\sum_{j=-N+l}^{N-1} \sum_{J=j-l}^{j-1}\left(u_{-}-u_{j}\right)+\sum_{j=N+1}^{N} \sum_{J=j-l}^{N-1}\left(u_{-}-u_{j}\right) \\
& =\sum_{-N+1}^{-N+l-1}(J+N-1)\left(u_{-}-u_{j}\right)+\sum_{j=-N+l}^{N} l\left(u_{-}-u_{j}\right)+\frac{l(l+1)}{2}\left(u_{-}-u_{+}\right) \\
& =\sum_{-N+1}^{-N+l-1}(J+N-1-l)\left(u_{-}-u_{j}\right)+\sum_{j=-N}^{N-1} l\left(u_{-}-u_{j}\right)+\frac{l(l+1)}{2}\left(u_{-}-u_{+}\right)
\end{aligned}
$$

Combining (4.3) and (4.4) with (4.2), we obtain

$$
\begin{aligned}
& \epsilon\left(u_{+}-u_{-}\right)=2 N \epsilon\left(u_{+}-u_{N-1}\right)+2 N \sum_{j=-m q}^{l}\left(u_{-N+j}^{m}-u_{-}\right) \\
& +\sum_{-N+1}^{-N+l-1}(J+N-1-l)\left(u_{-}-u_{j}\right)+\frac{l(l+1)}{2}\left(u_{-}-u_{+}\right) \\
& +\sum_{j=-N}^{N-1}\left[l\left(u_{-}-u_{j}\right)-\lambda \sum_{k=0}^{m-1}\left(f_{-}-\bar{f}_{j+l+1 / 2}^{k}\right)\right] \\
& =2 N \epsilon\left(u_{+}-u_{N-1}\right)+2 N \sum_{j=-m q}^{l}\left(u_{-N+j}^{m}-u_{-}\right) \\
& +\sum_{-N+1}^{-N+l-1}(J+N-1-l)\left(u_{-}-u_{j}\right)+\frac{l(l+1)}{2}\left(u_{-}-u_{+}\right) \\
& -\lambda \sum_{k=0}^{m-1} \sum_{j=0}^{l-1}\left(f_{-N+j+1 / 2}^{k}-\bar{f}_{N+j+1 / 2}^{k}\right)-\frac{\lambda l}{m} \sum_{k=0}^{m-1} \sum_{i=0}^{k-1}\left(\bar{f}_{N+1 / 2}^{i}-\bar{f}_{-N+1 / 2}^{i}\right) \\
& +\sum_{j=-N}^{N-1} \sum_{k=0}^{m-1}\left[\frac{l}{m}\left(u_{-}-u_{j}^{k}\right)-\lambda\left(f_{-}-\bar{f}_{j+1 / 2}^{k}\right)\right]
\end{aligned}
$$

where we used

$$
\begin{aligned}
& \sum_{j=-N+1}^{N}\left(u_{j}^{k}-u_{j}\right)=-\lambda \sum_{i=0}^{k-1} \sum_{j=-N+1}^{N}\left[\bar{f}_{j+1 / 2}^{i}-\bar{f}_{j-1 / 2}^{i}\right] \\
= & -\lambda \sum_{i=0}^{k-1}\left[\bar{f}_{N+1 / 2}^{i}-\bar{f}_{-N+1 / 2}^{i}\right] .
\end{aligned}
$$

Rearranging terms in (4.5), we arrive at (4.1). 
Corollary 4.2. Let $u_{j}$ be a decreasing solution of (1.10) with $-L=M=N>0$. Then $u_{j}$ satisfies

$$
-\sum_{j=-N}^{N-1} \sum_{k=0}^{m-1}\left[\frac{l}{m}\left(u_{-}-u_{j}^{k}\right)-\lambda\left(f_{-}-\bar{f}_{j+1 / 2}^{k}\right)\right] \leq C,
$$

where $C$ is bounded uniformly in $N$ and $\epsilon \in[0,1]$.

Proof. We note that the left hand side of (4.1), denoted as $C_{1}$, is bounded uniformly in $N$ and $\epsilon \in[0,1]$. Rearranging terms in (4.1), we obtain

$$
\begin{aligned}
& -\sum_{j=-N}^{N-1} \sum_{k=0}^{m-1}\left[\frac{l}{m}\left(u_{-}-u_{j}^{k}\right)-\lambda\left(f_{-}-\bar{f}_{j+1 / 2}^{k}\right)\right] \\
& =-C_{1}+2 N \epsilon\left(u_{+}-u_{N-1}\right)+2 N \sum_{j=-m q}^{l}\left(u_{-N+j}^{m}-u_{-}\right) .
\end{aligned}
$$

Since $u_{j}$ is decreasing and the scheme is monotonicity preserving, the terms $u_{+}-$ $u_{N-1} \leq 0, u_{-N+j}^{m}-u_{-} \leq 0$. The inequality (4.6) immediately follows.

Lemma 4.3. Let $u_{j}$ be a decreasing solution of (1.9). Then $u_{j}$ satisfies

$$
\begin{aligned}
& \sum_{j=-\infty}^{\infty} \sum_{k=0}^{m-1}\left[\frac{l}{m}\left(u_{-}-u_{j}^{k}\right)-\lambda\left(f_{-}-\bar{f}_{J+1 / 2}^{k}\right)\right] \\
& =-\frac{l}{2}(1-\lambda s)\left(u_{-}-u_{+}\right) .
\end{aligned}
$$

Proof. Apply the same technique used in the proof of Lemma 4.3 to (1.9), we can prove (4.7). Since we will not use this lemma in this paper, we omit the details of the proof.

\section{Monotonicity PRESERVIng SCHEMES With $C^{1}$ FLuX FunCtions HAVE TRAVELING WAVE SOLUTIONS}

In this section, we shall prove the existence of discrete shock profiles of monotonicity preserving schemes with $C^{1}$ flux functions $\bar{f}$ by verifying conditions (3.14) for these schemes.

Theorem 5.1. Monotonicity preserving schemes with $C^{1}$ flux functions $\bar{f}_{j+1 / 2}$ have decreasing traveling wave solutions when $\lambda$ s is rational.

Proof. To verify (3.14), we let $u_{j},|j| \leq N$ be a decreasing solution of (1.10) with $-L=M=N>0$. We consider the identity

$$
\begin{aligned}
& f_{-}-\bar{f}_{j+1 / 2}= \bar{f}\left(u_{-}, u_{-}, \ldots, u_{-}\right)-\bar{f}\left(u_{j-p+1}, u_{j-p+2}, \ldots, u_{j+q}\right) \\
&=\sum_{i=-p+1}^{q}\left(u_{-}-u_{j+i}\right) \int_{0}^{1} \bar{f}_{i}\left(u_{j-p+1}-\beta\left(u_{-}-u_{j-p+1}\right), \ldots, u_{j+q}\right. \\
&\left.+\beta\left(u_{-}-u_{j+q}\right)\right) d \beta
\end{aligned}
$$

where

$$
\bar{f}_{i}\left(u_{-p+1}, u_{-p+2}, \ldots, u_{q}\right):=\frac{\partial \bar{f}\left(u_{-p+1}, u_{-p+2}, \ldots, u_{q}\right)}{\partial u_{i}} .
$$


For simplicity, we use the following short notation in (5.1)

$$
F\left(i, j, u, u_{-}\right):=\int_{0}^{1} \bar{f}_{i}\left(u_{j-p+1}-\beta\left(u_{-}-u_{j-p+1}\right), \ldots, u_{j+q}+\beta\left(u_{-}-u_{j+q}\right)\right) d \beta
$$

to yield

$$
\begin{aligned}
& \sum_{j=-N}^{J}\left(f_{-}-\bar{f}_{j+1 / 2}\right)=\sum_{j=-N}^{J} \sum_{i=-p+1}^{q} F\left(i, j, u, u_{-}\right)\left(u_{-}-u_{j+i}\right) \\
& =\sum_{i=-p+1} \sum_{j=-N+i}^{J+i} F\left(i, j-i, u, u_{-}\right)\left(u_{-}-u_{j}\right) \\
& =\sum_{j=-N}^{J}\left(u_{-}-u_{j}\right) \sum_{i=-p+1}^{q} F\left(i, j-i, u, u_{-}\right)+C
\end{aligned}
$$

for any $-N \leq J \leq N$, where $C>0$ is a constant bounded uniformly in $N$ and $\epsilon>0$. We note that from the consistency condition (1.3), we have

$$
\sum_{i=-p+1}^{q} \bar{f}_{i}(w, w, \ldots, w)=f^{\prime}(w)
$$

for any constant $w$. Let $\bar{u}_{j}$ denote the arithmetic average of $u_{j-i+i_{0}}, i, i_{0}=-p+$ $1, \ldots, q$. By the uniform continuity of $\bar{f}$ when its variables are in any bounded set, for any constant $\gamma>0$, there is $\delta>0$ such that if

$$
\left|u_{j-i+i_{0}}-\bar{u}_{j}\right|<\delta
$$

for $i, i_{0}=-p+1, \ldots, q$, then

$$
\begin{aligned}
& \mid \sum_{i=-p+1}^{q} \bar{f}_{i}\left(u_{j-i-p+1}+\beta\left(u_{-}-u_{j-i-p+1}\right), \ldots, u_{j-i+q}+\beta\left(u_{-}-u_{j-i+q}\right)\right) \\
& \quad-f^{\prime}\left(\bar{u}_{j}+\beta\left(u_{-}-\bar{u}_{j}\right)\right) \mid<\gamma,
\end{aligned}
$$

and hence

$$
\begin{aligned}
& \left|\sum_{i=-p+1}^{q} F\left(i, j-i, u, u_{-}\right)-\int_{0}^{1} f^{\prime}\left(\bar{u}_{j}+\beta\left(u_{-}-\bar{u}_{j}\right)\right) d \beta\right| \\
& =\left|\sum_{i=-p+1}^{q} F\left(i, j-i, u, u_{-}\right)-\frac{f_{-}-f\left(\bar{u}_{j}\right)}{u_{-}-\bar{u}_{j}}\right|<\gamma .
\end{aligned}
$$


Similarly, we can prove that if (5.6) is satisfied, then

$$
\begin{aligned}
& \mid \sum_{i=-p+1}^{q} \bar{f}_{i}\left(u_{j-i-p+1}+\beta\left(u_{+}-u_{j-i-p+1}\right), \ldots, u_{j-i+q}+\beta\left(u_{+}-u_{j-i+q}\right)\right) \\
& \quad-f^{\prime}\left(\bar{u}_{j}+\beta\left(u_{+}-\bar{u}_{j}\right)\right) \mid<\gamma
\end{aligned}
$$

and hence

$$
\left|\sum_{i=-p+1}^{q} F\left(i, j-i, u, u_{+}\right)-\frac{f_{+}-f\left(\bar{u}_{j}\right)}{u_{+}-\bar{u}_{j}}\right|<\gamma
$$

We define the set of indices

$$
\mathcal{A}:=\{j \in \mathbb{Z}:-N \leq j \leq N \text { and (5.6) is satisfied }\}
$$

and

$$
\mathcal{B}:=\{i \in \mathbb{Z}:|i-j| \leq p+q+1 \text { for any } j \in[-N, N] \backslash \mathcal{A}\}
$$

In other words, $\mathcal{B}$ is the $p+q+1$ neighborhood of $\mathcal{A}^{c}$ in $\mathbb{Z} \cap[-N, N]$. Since $u_{j}$ is decreasing from $u_{-}$to $u_{+}$, the number of elements in $\mathcal{A}^{c}$ and hence that in $\mathcal{B}$ depend only on $\delta>0$.

Now, we consider

$$
\begin{aligned}
\sum_{j=-N}^{N}[ & \left.\frac{l}{m}\left(u_{-}-u_{j}\right)-\lambda\left(f_{-}-\bar{f}_{j+1 / 2}\right)\right] \\
= & \sum_{j=-N}^{J(u)-q-p} \chi(j \notin \mathcal{B})\left[\frac{l}{m}\left(u_{-}-u_{j}\right)-\lambda\left(f_{-}-\bar{f}_{j+1 / 2}\right)\right] \\
& +\sum_{j=J(u)+p+q}^{N} \chi(j \notin \mathcal{B})\left[\frac{l}{m}\left(u_{+}-u_{j}\right)-\lambda\left(f_{+}-\bar{f}_{j+1 / 2}\right)\right] \\
& +\sum_{j=-N}^{N} \chi(\mathcal{B} \text { or } j=J(u)-p-q+1, J(u)-p-q+2, \ldots, J(u)+p+q-1) \\
& \times\left[\frac{l}{m}\left(u--u_{j}\right)-\lambda\left(f_{-}-\bar{f}_{j+1 / 2}\right)\right] \\
= & : I+I I+I I I,
\end{aligned}
$$

where

$$
\chi(A)= \begin{cases}1, & \text { if } A \text { is true } \\ 0, & \text { if } A \text { is false }\end{cases}
$$


The term $I I I$ of (5.11) is bounded uniformly in $N$ and $\epsilon \in(0,1)$ since the number of terms in III only depends on $\delta, p$ and $q$. The term $I$ can be estimated as follows:

$$
\begin{aligned}
I: & \sum_{j=-N}^{J(u)-q-p} \chi(j \notin \mathcal{B})\left[\frac{l}{m}\left(u_{-}-u_{j}\right)-\lambda\left(f_{-}-\bar{f}_{j+1 / 2}\right)\right] \\
& \sum_{j=-N}^{J(u)-q-p} \chi(j \notin \mathcal{B}) \frac{l}{m}\left(u_{-}-u_{j}\right) \\
& -\sum_{j=-N}^{J(u)-q-p} \chi(j \notin \mathcal{B}) \lambda\left(f_{-}-\bar{f}_{j+1 / 2}\right) \\
& =\sum_{j=-N}^{J(u)-q-p} \chi(j \notin \mathcal{B}) \frac{l}{m}\left(u_{-}-u_{j}\right) \\
& -\sum_{j=-N}^{J(u)-q-p} \chi(j \notin \mathcal{B})\left(u_{-}-u_{j}\right) \sum_{i=-p+1}^{q} F\left(i, j-i, u, u_{-}\right)+C,
\end{aligned}
$$

where in the last step we used (5.4) and the number $C$ includes the $C$ from (5.4) and finitely many other terms of the form $\left(u_{-}-u_{k}\right) F\left(i, j, u, u_{-}\right)$. This number $C$ is bounded uniformly in $N$ and $\epsilon>0$. Applying (5.8) in the last sum of the above, we get

$$
I \leq-\lambda \sum_{j=-N}^{J(u)-q-p} \chi(j \notin \mathcal{B})\left(-s+\frac{f_{-}-f\left(\bar{u}_{j}\right)}{u_{-}-\bar{u}_{j}}-\gamma\right)\left(u_{-}-u_{j}\right)+C .
$$

Note that the range of $\bar{u}_{j}$ in above sum is in $\left[\left(u_{+}+u_{-}\right) / 2, u_{-}\right]$. By the chord condition (1.8), the constant

$$
\begin{array}{r}
\alpha:=\min \left[\min _{\bar{u} \in\left[\left(u_{+}+u_{-}\right) / 2, u_{-}\right]}\left(-s+\frac{f_{-}-f(\bar{u})}{u_{-}-\bar{u}}\right),\right. \\
\left.\min _{\bar{u} \in\left[u_{+},\left(u_{+}+u_{-}\right) / 2\right]}\left(s-\frac{f_{+}-f(\bar{u})}{u_{+}-\bar{u}}\right)\right]>0 .
\end{array}
$$

Recalling $\gamma>0$ is arbitrarily chosen, we can let $0<\gamma<\alpha / 2$. This choice of $\gamma$ leads to

$$
I \leq-\frac{\lambda \alpha}{2} \sum_{j=-N}^{J(u)-q-p} \chi(j \notin \mathcal{B})\left|u_{j}-u_{-}\right|+C
$$

Similarly, we have

$$
I I \leq-\frac{\lambda \alpha}{2} \sum_{j=J(u)+q+p} \chi(j \notin \mathcal{B})\left|u_{j}-u_{+}\right|+C .
$$


Combining (5.11)-(5.14), we prove that

$$
\begin{aligned}
& \sum_{j=-N}^{N}\left[\frac{l}{m}\left(u_{-}-u_{j}\right)-\lambda\left(f_{-}-\bar{f}_{j+1 / 2}\right)\right] \\
& \leq-\frac{\lambda \alpha}{2} \sum_{j=-N}^{N}\left|u_{j}-V_{j}\right|+C,
\end{aligned}
$$

where $C>0$ is a constant bounded uniformly in $N$ and $\epsilon>0$. The arguments above and hence (5.15) remain valid if we replace all $u$ and $v$ by $u^{k}$ and $v^{k}$. Plugging (5.15) into (4.6) yields

$$
\sum_{j=-N}^{N}\left|u_{j}-V_{j}\right| \leq C,
$$

which is desired.

\section{ExistenCE OF DisCRETE SHOCK PROFILES FOR THE SCHEME (1.3)}

In this section, we shall prove the existence of traveling waves for second order upwinding, with $f^{\prime}>0$, flux based MUSCL scheme (1.3) by verifying that it satisfies the condition (3.14).

Lemma 6.1. The function $\hat{f}_{j+1 / 2}$ defined in (1.3b) satisfies

$$
\min \left(f\left(u_{j}\right), f\left(u_{j \pm 1}\right)\right) \leq \hat{f}_{j+1 / 2}(u) \leq \max \left(f\left(u_{j}\right), f\left(u_{j \pm 1}\right)\right) .
$$

Proof. For simplicity, we use the notation $\delta_{j} f:=f\left(u_{j+1}\right)-f\left(u_{j}\right)$ and $\delta_{j} f^{k}:=$ $f\left(u_{j+1}^{k}\right)-f\left(u_{j}^{k}\right)$ throughout this paper. According to the definition (1.3b) for $\hat{f}$, there are three cases:

Case 1. $\delta_{j} f \delta_{j-1} f>0$ and $\left|\delta_{j} f\right| \leq\left|\delta_{j-1} f\right|$. In this case,

$$
\hat{f}_{j+1 / 2}=\left(f\left(u_{j+1}\right)+f\left(u_{j}\right)\right) / 2,
$$

and hence (3.4) holds.

Case 2. $\delta_{j} f \delta_{j-1} f>0$ and $\left|\delta_{j} f\right| \geq\left|\delta_{j-1} f\right|$. In this case, we have $\hat{f}_{j+1 / 2}=$ $f\left(u_{j}\right)+\delta_{j-1} f / 2$. If $\delta_{j-1} f<0$, then

$$
f\left(u_{j}\right)>f\left(u_{j}\right)+\delta_{j-1} f / 2 \geq f\left(u_{j}\right)+\delta_{j} f / 2 \geq \min \left(f\left(u_{j}\right), f\left(u_{j \pm 1}\right)\right) .
$$

If $\delta_{j-1} f>0$, then

$$
\begin{aligned}
& \min \left(f\left(u_{j}\right), f\left(u_{j \pm 1}\right)\right) \leq f\left(u_{j}\right)<f\left(u_{j}\right)+\delta_{j-1} f / 2 \\
& \leq f\left(u_{j}\right)+\delta_{j} f / 2 \leq \max \left(f\left(u_{j}\right), f\left(u_{j \pm 1}\right)\right) .
\end{aligned}
$$

In either cases lead to (3.4).

Case 3. $\delta_{j} f \delta_{j-1} f \leq 0$. Then, $\hat{f}_{j+1 / 2}=f\left(u_{j}\right)$ which obviously satisfies (6.1). 
Corollary 6.2. Let $u_{j}$ be a decreasing function and $f^{\prime}>0$. Then $f\left(u_{j+1}\right) \leq$ $\hat{f}_{j+1 / 2}(u) \leq f\left(u_{j-1}\right)$.

Theorem 6.3. The second order upwinding flux based MUSCL scheme (1.3) has a traveling wave when $\lambda s$ is rational.

Proof. By Theorem 3.5 it suffices to prove (3.14) for decreasing solutions $u_{j}$ of (1.10). We start with (4.6):

$$
\sum_{k=0}^{m-1} \sum_{j=-N+1}^{N}\left[\frac{l}{m}\left(u_{+}-u_{j}\right)-\lambda\left(f_{+}-\bar{f}_{j+l-1 / 2}^{k}\right)\right] \geq C,
$$

which holds for decreasing solutions of (1.10). From (1.10c), we have

$$
\begin{aligned}
& \sum_{j=-N+1}^{N}\left(u_{j}^{k}-u_{j}\right)=-\lambda \sum_{i=0}^{k-1} \sum_{j=-N+1}^{N}\left[\bar{f}_{j+1 / 2}^{i}-\bar{f}_{j-1 / 2}^{i}\right] \\
= & -\lambda \sum_{i=0}^{k-1}\left[\bar{f}_{N+1 / 2}^{i}-\bar{f}_{-N+1 / 2}^{i}\right] \leq-\lambda k\left(f_{+}-f_{-}\right)
\end{aligned}
$$

and

$$
\begin{aligned}
& \sum_{j=-N+1}^{N}\left(u_{j}^{k+1 / 2}-u_{j}\right) \\
& \quad=-\lambda \sum_{i=0}^{k-1} \sum_{j=-N+1}^{N}\left[\bar{f}_{j+1 / 2}^{i}-\bar{f}_{j-1 / 2}^{i}\right]+\sum_{j=-N+1}^{N}\left[\hat{f}_{j+1 / 2}^{k}-\hat{f}_{j-1 / 2}^{k}\right] \\
& \quad \leq-\lambda(k+1)\left(f_{+}-f_{-}\right) .
\end{aligned}
$$

Applying (6.3) to (6.2), we obtain

$$
\begin{aligned}
C & \leq \sum_{k=0}^{m-1} \sum_{j=-N+1}^{N}\left[\frac{l}{m}\left(u_{+}-u_{j}\right)-\lambda\left(f_{+}-\bar{f}_{j+1 / 2}^{k}\right)\right] \\
\leq & \sum_{k=0}^{m-1} \sum_{j=-N+1}^{N}\left[\frac{l}{m}\left(u_{+}-\frac{u_{j}^{k}+u_{j}^{k+1 / 2}}{2}\right)-\frac{\lambda}{2}\left(2 f_{+}-\hat{f}_{j+1 / 2}^{k}-\hat{f}_{j+1 / 2}^{k+1 / 2}\right)\right] \\
& \frac{l m}{2} \lambda\left(f_{-}-f_{+}\right) \\
\leq & \frac{\lambda}{2} \sum_{k=0}^{m-1} \sum_{j=-N+1}^{N}\left[s\left(u_{+}-u_{j}^{k}\right)-\left(f_{+}-\hat{f}_{j+1 / 2}^{k}\right)\right] \\
& +\frac{\lambda}{2} \sum_{k=0}^{m-1} \sum_{j=-N+1}^{N}\left[s\left(u_{+}-u_{j}^{k+1 / 2}\right)-\left(f_{+}-\hat{f}_{j+1 / 2}^{k+1 / 2}\right)\right]+\frac{l m}{2} \lambda\left(f_{-}-f_{+}\right) \\
& =I+I I+\frac{l m}{2} \lambda\left(f_{-}-f_{+}\right) .
\end{aligned}
$$


Using Lemma 6.1 and that $u_{j}$ and hence $u_{j}^{k}$ and $u_{j}^{k+1 / 2}$ are decreasing and $f^{\prime}>0$, we can estimate the term $I$ in (6.4) as

$$
\begin{aligned}
& 2 I / \lambda:=\sum_{k=0}^{m-1} \sum_{j=-N+1}^{N}\left[s\left(u_{+}-u_{j}^{k}\right)-\left(f_{+}-\hat{f}_{j+1 / 2}^{k}\right)\right] \\
& =\sum_{k=0}^{m-1} \sum_{j=-N+1}^{N}\left[s\left(u_{+}-u_{j}^{k}\right)-\left(f_{+}-\hat{f}_{j+3 / 2}^{k}\right)\right]+\sum_{k=0}^{m-1}\left[f_{N+3 / 2}^{k}-f_{-N+1 / 2}^{k}\right] \\
& \leq \sum_{k=0}^{m-1} \sum_{j=-N+1}^{N}\left[s\left(u_{+}-u_{j}^{k}\right)-\left(f_{+}-\hat{f}\left(u_{j}^{k}\right)\right]+C_{1}\right. \\
& =\sum_{k=0}^{m-1}\left\{\sum _ { j = - N + 1 } ^ { J ( u ^ { k } ) } \left[s\left(u_{-}-u_{j}^{k}\right)-\left(f_{-}-f\left(u_{j}^{k}\right)\right]\right.\right. \\
& +\sum_{j=J\left(u^{k}\right)}^{N}\left[s\left(u_{+}-u_{j}^{k}\right)-\left(f_{+}-f\left(u_{j}^{k}\right)\right]\right\}+C_{1} \\
& \leq-\alpha \sum_{k=0}^{m-1} \sum_{j=-N+1}^{N}\left|u_{j}^{k}-V_{j}^{k}\right|+C_{1},
\end{aligned}
$$

where in the last step we used (5.12). The number $C_{1}$ is bounded uniformly in $N$ and $\epsilon \in[0,1]$. Similarly, we can prove that

$$
\begin{aligned}
& 2 I I / \lambda:=\sum_{k=0}^{m-1} \sum_{j=-N+1}^{N}\left[s\left(u_{+}-u_{j}^{k+1 / 2}\right)-\left(f_{+}-\hat{f}_{j+1 / 2}^{k+1 / 2}\right)\right] \\
& \leq-\alpha \sum_{k=0}^{m-1} \sum_{j=-N+1}^{N}\left|u_{j}^{k+1 / 2}-V_{j}^{k+1 / 2}\right|+C_{2} .
\end{aligned}
$$

The desired inequality (3.14) follows immediately from (6.4) and (6.5).

\section{Existence of TRAVELING WAVES For MUSCL SCheme (1.4)}

In this section, we shall prove the existence discrete traveling waves of MUSCL scheme (1.4). It follows from Theorem 2.3 that there is a decreasing solution of (1.9) for MUSCL scheme (1.4).

From (1.4a) we see that

$$
u_{j}^{1}=u_{j}-\frac{\lambda}{2}\left(\hat{f}_{j+1 / 2}+\hat{f}_{j+1 / 2}^{1 / 2}-\hat{f}_{j-1 / 2}-\hat{f}_{j-1 / 2}^{1 / 2}\right),
$$

where $\hat{f}_{j+1 / 2}^{1 / 2}=\hat{f}_{j+1 / 2}\left(u^{1 / 2}\right)$. This infers the expression for $\bar{f}$ :

$$
\bar{f}_{j+1 / 2}=\frac{1}{2}\left(\hat{f}_{j+1 / 2}+\hat{f}_{j+1 / 2}^{1 / 2}\right) .
$$

Theorem 7.1. The MUSCL scheme (1.4) has a decreasing traveling wave solution when $\lambda$ s is rational.

Proof. It suffices to prove that any decreasing solution of (1.10) for the MUSCL scheme (1.4) satisfies (3.14). 
In the sequel, we use the notation $f_{ \pm}^{-}=f^{-}\left(u_{ \pm}\right)$and $f_{ \pm}^{+}=f^{+}\left(u_{ \pm}\right)$. We start with (4.6) without writing down $\sum_{k=0}^{m-1}$ and index $k$ :

$$
\begin{aligned}
C & \leq \sum_{j=-N}^{N}\left[\frac{l}{m}\left(u_{-}-u_{j}\right)-\lambda\left(f_{-}-\bar{f}_{j+1 / 2}\right)\right] \\
= & \sum_{j=-N}^{N}\left[\frac{l}{m}\left(u_{-}-\frac{u_{j}+u_{j}^{1 / 2}}{2}\right)-\lambda\left(f_{-}-\bar{f}_{j+1 / 2}\right)\right]+\frac{\lambda l}{m}\left(\hat{f}_{N+1 / 2}-\hat{f}_{-N-1 / 2}\right) \\
= & \lambda \sum_{j=-N}^{N}\left[\frac{s-a}{2}\left(u_{-}-\frac{u_{j}+u_{j}^{1 / 2}}{2}\right)-\left(f_{-}^{-}-\frac{\hat{f}_{j+1 / 2}^{-}(u)+\hat{f}_{j+1 / 2}^{-}\left(u^{1 / 2}\right)}{2}\right)\right] \\
+ & \lambda \sum_{j=-N}^{N}\left[\frac{s+a}{2}\left(u_{-}-\frac{u_{j}+u_{j}^{1 / 2}}{2}\right)-\left(f_{-}^{+}-\frac{\hat{f}_{j+1 / 2}^{+}(u)+\hat{f}_{j+1 / 2}^{+}\left(u^{1 / 2}\right)}{2}\right)\right]+O(1) \\
= & I+I I+O(1),
\end{aligned}
$$

where $O(1)$ is bounded independent of $N$ and $\epsilon \in[0,1]$. We recall that Lemma 6.1 holds for any $f$. Since $u_{j}$ is decreasing and $\left(f^{-}\right)^{\prime}<0$, Lemma 6.1 implies that

$$
f^{-}\left(u_{j+1}\right) \geq \hat{f}_{j+1 / 2}^{-} \geq f^{-}\left(u_{j-1}\right) .
$$

The first term $I$ in (7.2) can be estimated as follows:

$$
\begin{aligned}
I / \lambda:=\sum_{j=-N}^{N}\left[\frac{s-a}{2}\left(u_{-}-\frac{u_{j}+u_{j}^{1 / 2}}{2}\right)\right. \\
\left.-\left(f_{-}^{-}-\frac{\hat{f}_{j+1 / 2}^{-}(u)+\hat{f}_{j+1 / 2}^{-}\left(u^{1 / 2}\right)}{2}\right)\right] \\
\leq \frac{1}{2} \sum_{j=-N}^{N}\left[\frac{s-a}{2}\left(u_{-}-u_{j}\right)-\left(f_{-}^{-}-f^{-}\left(u_{j+1}\right)\right)\right] \\
+\frac{1}{2} \sum_{j=-N}^{N}\left[\frac{s-a}{2}\left(u_{-}-u_{j}^{1 / 2}\right)-\left(f_{-}^{-}-f^{-}\left(u_{j+1}^{1 / 2}\right)\right)\right] .
\end{aligned}
$$

By the chord condition (1.8), the constant

$$
\begin{aligned}
& \alpha:=\min \left[\min _{\bar{u} \in\left[\left(u_{+}+u_{-}\right) / 2, u_{-}\right]}\left(-(s \pm a)+\frac{f^{p} m_{-}-f^{ \pm}(\bar{u})}{u_{-}-\bar{u}}\right),\right. \\
& \left.\min _{\bar{u} \in\left[u_{+},\left(u_{+}+u_{-}\right) / 2\right]}\left((s \pm a)-\frac{f_{+}^{ \pm}-f^{ \pm}(\bar{u})}{u_{+}-\bar{u}}\right)\right]>0 .
\end{aligned}
$$


Applying (7.4) to (7.3) we get

$$
I / \lambda \leq-\frac{\alpha}{2} \sum_{j=-N}^{N}\left(\left|u_{j}-V_{j}\right|+\left|u^{1 / 2}-V_{j}^{1 / 2}\right|\right)+\left|f_{-}^{-}-f_{+}^{-}\right|,
$$

where $V_{j}^{1 / 2}$ is defined in (1.11) with $u$ replaced by $u^{1 / 2}$. Similarly, we can prove that

$$
I I / \lambda \leq-\frac{\alpha}{2} \sum_{j=-N}^{N}\left(\left|u_{j}-V_{j}\right|+\left|u_{j}^{1 / 2}-V_{j}^{1 / 2}\right|\right)+\left|f_{-}^{+}-f_{+}^{+}\right| .
$$

Then, inequality (3.14) follows from (7.2), (7.5) and (7.6).

\section{Existence of traveling Waves for the Godunov scheme}

The existence of traveling waves of the Godunov scheme can also be proved using Theorems 3.5 and Corollary 4.2.

Theorem 8.1. The Godunov scheme has a decreasing traveling wave solution when $\lambda s$ is rational.

Proof. Let $u_{j}$ be a decreasing solution of (1.10). For decreasing $u_{j}$, the numerical flux function for the Godunov scheme is

$$
\bar{f}_{j+1 / 2}=\max \left(f\left(u_{j}\right), f\left(u_{j+1}\right)\right)
$$

By the transformation $x \mapsto x-c t$ if necessary, we can assume, without loss of generality, that $f^{\prime}\left(\left(u_{+}+u_{-}\right) / 2\right)=0$. Under this assumption, the expression (8.1) for decreasing $u_{j}$ is

$$
\begin{gathered}
\bar{f}_{j+1 / 2}=\max \left(f\left(u_{j}\right), f\left(u_{j+1}\right)\right) \\
\left(8.1^{\prime}\right)= \begin{cases}f\left(u_{j}\right), & \text { if } u_{j} \geq\left(u_{+}+u_{-}\right) / 2 \text { and } u_{j+1} \geq\left(u_{+}+u_{-}\right) / 2, \\
f\left(u_{j+1}\right), & \text { if } u_{j} \leq\left(u_{+}+u_{-}\right) / 2 \text { and } u_{j+1} \leq\left(u_{+}+u_{-}\right) / 2, \\
\max \left(f\left(u_{j}\right), f\left(u_{j+1}\right)\right), & \text { else. }\end{cases}
\end{gathered}
$$

Again, we start verifying (3.14) with Corollary 4.2 by considering

$$
C \leq \sum_{k=0}^{m-1} \sum_{j=-N}^{N}\left[\frac{l}{m}\left(u_{-}-u_{j}\right)-\lambda\left(f_{-}-\bar{f}_{j+1 / 2}^{k}\right)\right] .
$$


For simplicity and without loss of generality, we omit the index $k$ in the following. From (8.1), we see that (8.2) can be further written as

$$
\begin{aligned}
& C \leq \sum_{j=-N}^{N}\left[\frac{l}{m}\left(u_{-}-u_{j}\right)-\lambda\left(f_{-}-\bar{f}_{j+1 / 2}\right)\right] \\
& =\sum_{|j| \leq N \text { and } \bar{f}_{j+1 / 2}=f\left(u_{j}\right)}\left[\frac{l}{m}\left(u_{-}-u_{j}\right)-\lambda\left(f_{-}-f\left(u_{j}\right)\right)\right] \\
& +\sum_{|j| \leq N \text { and } \bar{f}_{j+1 / 2}=f\left(u_{j+1}\right)}\left[\frac{l}{m}\left(u_{-}-u_{j}\right)-\lambda\left(f_{-}-f\left(u_{j+1}\right)\right)\right] \\
& =\sum_{|j| \leq N \text { and } \bar{f}_{j+1 / 2}=f\left(u_{j}\right)}\left[\frac{l}{m}\left(u_{-}-u_{j}\right)-\lambda\left(f_{-}-f\left(u_{j}\right)\right)\right] \\
& +\sum_{|j| \leq N \text { and } \bar{f}_{j+1 / 2}=f\left(u_{j+1}\right)}\left[\frac{l}{m}\left(u_{-}-u_{j+1}\right)-\lambda\left(f_{-}-f\left(u_{j+1}\right)\right)\right] \\
& +\sum_{|j| \leq N \text { and } \bar{f}_{j+1 / 2}=f\left(u_{j+1}\right)} \frac{l}{m}\left(u_{j+1}-u_{j}\right) .
\end{aligned}
$$

The last sum in (8.3) is bounded uniformly in $N$ and $\epsilon \in[0,1]$ since $u_{j}$ is monotone. Using the same method for obtaining (6.5), we can further manipulate the first two sums in the right hand side of (8.3) to yield

$$
\begin{aligned}
C \leq-\alpha \lambda & \left(\sum_{|j| \leq N \text { and } \bar{f}_{j+1 / 2}=f\left(u_{j}\right)}\left|u_{j}-V_{j}\right|\right. \\
& +\sum_{|j| \leq N} \text { and } \bar{f}_{j+1 / 2}=f\left(u_{j+1}\right) \\
& \left.\left|u_{j+1}-V_{j+1}\right|\right) .
\end{aligned}
$$

Combining the two summations in (8.4), we can rewrite it as

$$
\sum_{j=-N}^{N} b_{j}\left|u_{j}-V_{j}\right| \leq C
$$

where

$$
b_{j}= \begin{cases}0, & \text { if } \bar{f}_{j+1 / 2}=f\left(u_{j+1}\right) \text { and } \bar{f}_{j-1 / 2}=f\left(u_{j-1}\right) \\ 2, & \text { if } \bar{f}_{j+1 / 2}=\bar{f}_{j-1 / 2}=f\left(u_{j}\right) \\ 1, & \text { else }\end{cases}
$$


In view of $\left(8.1^{\prime}\right)$, there is at most one point $j$ where $b_{j}=0$. Thus, the inequality (8.5) implies

$$
\begin{aligned}
C & \geq \sum_{j=-N}^{N} b_{j}\left|u_{j}-V_{j}\right| \\
& =\sum_{j=-N}^{J(u)} b_{j}\left|u_{j}-u_{-}\right|+\sum_{j=J(u)+1}^{N} b_{j}\left|u_{j}-u_{+}\right| \\
& \geq \sum_{j=-N}^{N}\left|u_{j}-V_{j}\right|-\left|u_{+}-u_{-}\right| .
\end{aligned}
$$

Therefore (3.14) holds for the Godunov scheme.

Since the Godunov scheme is $l^{1}$-contracting, which implies monotonicity preserving, the results on the existence of discrete shock profiles for the Godunov scheme can be much stronger (see $[\mathrm{F}]$ ).

\section{ACKNowledgments}

I would like to thank Professor Chi-Wang Shu at Brown University and Dr. Shih-Hsien Yu for many discussions.

\section{REFERENCES}

[EY] B. Engquist and Shih-Hsien Yu, Convergence of Lax-Wendroff scheme for piecewise smooth solutions with shocks, IMA preprint, (1995)

[F] Haitao Fan, Existence and uniquensss of traveling waves and error estimates for Godunov schemes of conservation laws, Math. Comp. 67 (1998) 87-109. MR 98h:65040

[Je] G. Jennings, Discrete shocks, Comm. Pure Appl. Math., 27 (1974) 25-37. MR 49:3358

[LX] Jiang-Guo Liu and Zhouping Xin, Nonlinear stability of discrete shocks for systems of conservation laws, Arch. Rational Mech. Anal., 125 (1993) 217-256. MR 95c:35166

[Ma] M. Mahwin, Topological degree methods in nonlinear boundary value problems, in CBMS Regional Conference Series in Mathematics, Am. Math. Soc., Vol. 40, Providence, 1979.

[Mi] D. Michelson, Discrete shocks for difference approximations to systems of conservation laws. Adv. Appl. Math., 5 (1984), 433-469. MR 86f:65159

[MR] A. Majda and J. Ralston, Discrete shock profiles for systems of conservation laws, Comm. Pure Appl. Math., 32 (1979) 445-482. MR 81i:35108

[TT] Tao Tang and Zhen-Huan Teng, The sharpness of Kuznetsov's $O(\sqrt{\delta x}) L^{1}$-error estimate for monotone difference schemes, Math. Comp. 64 (1995), 581-589. MR 95f:65176

[TZ] Zhen-Huan Teng and Pingwen Zhang, Optimal $L^{1}$-rate of convergence for the viscosity method and monotone scheme to piecewise constant solutions with shocks, SIAM J. Numer. Anal. 34 (1997), no. 3, 959-978. MR 98f:65094

[Yu Shih-Hsien Yu, Existence of discrete shock profiles for the Lax-Wendroff scheme, preprint, 1995.

Department of Mathematics, Georgetown University, Washington, DC 20057

E-mail address: fan@archimedes.math.georgetown.edu 